\title{
La reducción en la fenomenología de J.-L. Marion
}

\section{Reduction in J.-L. Marion's phenomenology}

\author{
Jorge Luis Roggero*
}

Resumen: ¿Cuál es el estatuto de la reducción en la obra de Marion? Este artículo se propone aclarar esta operación fundamental en la fenomenología de la donación estableciendo su relación con la epoché entendida heideggerianamente como tonalidad afectiva fundamental.

Palabras clave: reducción, epoché, tonalidad afectiva fundamental, Heidegger, Patočka

Abstract: Which is the statute of reduction in Marion's work? This article aims to clarify this fundamental operation in the phenomenology of donation by establishing its relationship with the epoche understood in the Heideggerian way of a fundamental attunement.

Keywords: reduction, epoché, fundamental attunement, Heidegger, Patočka

No bay fenomenología que no pase por la "reducción"

Eugen Fink ${ }^{1}$

Como bien destaca Rudolf Bernet, en su ya célebre La vie du sujet, a partir de 1990, con la obra de Marion, con la publicación de Phénoménologie matérielle de

\footnotetext{
* Abogado y licenciado en Filosofía de la Universidad de Buenos Aires. Doctor en Filosofía por la Universidad de Buenos Aires y por la Universidad de Paris IVSorbona. Becario post-doctoral del CONICET. Investigador asociado de los Archivos Husserl de Paris (ENS), del CLAFEN y de la Academia Nacional de Ciencias de Buenos Aires. Ha publicado libros, capítulos de libros y artículos en sus áreas de especialidad. Dirección electrónica: jorgeluisroggero@gmail.com

1 Fink, Eugen, "Die phänomenologische Philosophie Edmund Husserls in der gegenwärtigen Kritik", Kant-Studien, 38, 1-2 (1933), p. 342, n. 1.
} 
Michel Henry y de su comentario sobre los principios de la fenomenología, y con la publicación Heidegger et la phénoménologie de Jean-François Courtine, se abre en Francia un debate en torno a la reducción y el método fenomenológico. ${ }^{2}$ ¿Es la reducción una operación fundamental para la fenomenología o puede prescindirse de ella? Este debate aun reviste actualidad principalmente porque la obra de Marion se encuentra en pleno vigor $\mathrm{y}$ ha despertado un profundo interés en investigadores de la fenomenología de diversas partes del mundo.

En Reprise du donné, publicado en 2016, Marion refiere al cuestionamiento de la reducción en la escena francesa contemporánea, en autores como Claude Romano, Dominique Pradelle y Jocelyn Benoist. Según el autor, estos planteos no son novedosos: no sólo encuentran un temprano antecedente en los discípulos de Gotinga, sino que se inscriben en una cierta tendencia histórica entre los fenomenólogos a rechazar o, al menos, a atenuar la reducción. Tal es el caso de Maurice Merleau-Ponty, Hans-Georg Gadamer y Paul Ricœur. ${ }^{3}$

La posición de Marion es muy particular porque si bien defiende la necesidad de aplicar la reducción como método propio de la fenomenología, ${ }^{4}$ ciertamente no la entiende en el sentido husserliano de la reducción trascendental que reconduce a las operaciones de constitución y dación de sentido por parte de la conciencia, ni tampoco en el sentido heideggeriano de una reconducción del ente al ser. Pero ¿en qué sentido la entiende entonces? ¿No acierta Claude Romano al señalar que existe una tensión irreconciliable entre un tema asubjetivo (la noción heideggeriana de automostración del fenómeno) y una metodología subjetiva (la reducción)?5

2 Cfr. Bernet, Rudolf, La vie du sujet. Recherches sur l'interprétation de Husserl dans la phénoménologie, Paris, PUF, 1994, p. 5.

${ }^{3}$ Cfr. Marion, Jean-Luc, Reprise du donné, Paris, PUF, 2016, pp. 20-21.

${ }^{4}$ Marion afirma que aun cuando no exista una "vía regia" para la fenomenología ni tampoco para la filosofía -como bien destaca el propio Husserl-, "la reducción y su práctica no son opcionales (...) toda iniciativa fenomenológica se juega (o se pierde) en la puesta en práctica de la reducción” (Ibid., p. 19).

5 "Creo que hay una extraña paradoja en su libro al querer mantener, cueste lo que cueste, este método [la reducción] que va rigurosamente en contra lo que usted quiere mostrar. En efecto, si su objetivo es sacar a la luz el 'sí' del fenómeno y dejarle que 
Este artículo se propone clarificar la noción marioniana de reducción sosteniendo como hipótesis que para encontrar una respuesta es necesario advertir el modo en que se articula la reducción con la epoché y con "las tonalidades afectivas fundamentales" (Grundstimmungen). La especificidad propia de su concepción puede encontrarse en dos rasgos. El primero es la radicalización de la transformación heideggeriana del estatuto de la epoché, que pasa de ser un acto reflexivo a estar dada en una "experiencia fundamental" (Grunderfahrung), bajo la modalidad de una "tonalidad afectiva fundamental" (Grundstimmung). Marion va a señalar las limitaciones del temple de la angustia y va a explorar las posibilidades del aburrimiento y, finalmente, del amor. Esta última cuestión no está expresada de modo explícito y claro por Marion en estos términos, pero sostengo como hipótesis que puede leerse en Le phénomène érotique.

El segundo rasgo tiene que ver con la importancia decisiva que adquiere la epoché en el marco de la reducción. En este punto seguiremos a Jan Patočka y a la lectura del cuarto principio marioniano propuesta por Émilie Tardivel con base en ideas patočkianas. Patočka entiende que la epoché es el núcleo de la reducción ${ }^{6}$ y que deberían extremarse sus pretensiones poniendo entre paréntesis también al ego transcendental que la opera. ${ }^{7}$ Apoyándose en estas ideas, Émilie Tardivel afirma que la radicalización de la reducción llevada a cabo por Marion es en rigor una radicalización de la epoché; el cuarto principio debería enunciarse del siguiente modo: "A tanta epoché, tanta donación". 8

tome la iniciativa, si su objetivo es liberarlo de toda instancia trascendental que proporcionaría la medida de su fenomenicidad, ¿por qué apelar a un procedimiento que designa el método trascendental por excelencia, aquel por el cual el sujeto asegura su derecho sobre la fenomenicidad reconduciéndola a prestaciones constitutivas?". (Romano, Claude, "Remarques sur la méthode phénoménologique dans Étant donné de Jean-Luc Marion", Annales de philosopbie, 21 (2000), p. 11).

"Patočka, Jan, “¿Qué es la fenomenología?" en Jan Patočka, El movimiento de la existencia bumana, trad. T. Padilla, J. M. Ayuso y A. Serrano de Haro, Madrid, Ediciones Encuentro, 2004, p. 272.

7 Patočka, Jan, "Epoché y reducción" en Jan Patočka, El movimiento de la existencia bumana, p. 247.

8 Tardivel, Émilie, "Monde et donation. Une révision du quatrième principe de la phénoménologie", Revue de Métaphysique et de Morale, 85, 1 (2015), p. 125. 
Sostengo que el principio propuesto por Marion ("a tanta reducción, tanta donación"), efectivamente, implica la universalización de la epoché ("a tanta epoché, tanta donación"), pero en este gesto no se elimina la reducción ni se prescinde de ella, sino que se la potencia al máximo al permitir la reconducción de todo fenómeno a la instancia originaria de su donación. En este sentido, afirmo que en la fenomenología marioniana se da un solapamiento entre epoché y reducción: la epoché asume la función de la reducción de reconducción a lo originario en su operación de puesta entre paréntesis.

Bajo la influencia heideggeriana, Marion desplaza la epoché al campo no teórico al entenderla como operada por un temple anímico. Pero -al modo patočkiano-, Marion extrema la epoché, la generaliza. Buscando una radicalidad mayor a la de la angustia, Marion propone como disposición afectiva fundamental al aburrimiento y al amor. Y esta ampliación permite que la suspensión de la epoché devenga ella misma una reconducción. Como bien destaca Michel Henry, la reducción marioniana (podríamos agregar: operando como una epoché radicalizada) constituye una restitución de la reducción a su sentido positivo, pues ella ya no limita, ni restringe ni omite, sino que "abre y da [ouvre et donne]". 9

Con el fin de demostrar estas afirmaciones seguiré los siguientes pasos argumentativos. En un primer apartado recordaré brevemente la distinción entre las nociones de reducción y epoché tal como debe ser leída en la obra de Husserl. Esto nos permitirá entender la función de cada operación y el modo en que pueden articularse. En un segundo apartado, me detendré en el modo en que Marion concibe la epoché como temple anímico fundamental. Con este objetivo, será necesario dar cuenta de la impronta decisiva que deja Heidegger en la concepción marioniana. En un tercer apartado expondré la compleja relación entre epoché y reducción en la obra de Marion, en diálogo con la idea de universalización de la epoché presentada por Patočka. Finalmente, extraeré algunas conclusiones.

\section{La distinción entre epoché y reducción en Husserl}

\footnotetext{
${ }^{9}$ Henry, Michel, "Quatre principes de la phénoménologie", Revue de Métaphysique et de Morale. À propos de Réduction et donation de Jean-Luc Marion, 96, 1 (1991), p. 13.
} 
Como es bien sabido, la teoría husserliana de la reducción comienza a desarrollarse a partir de 1905 y se despliega en algunos textos clave de la obra de Husserl: Die Idee der Phänomenologie (1907), Philosophie als strenge Wissenschaft (1910/1911); Ideen I (1913), el segundo volumen del curso Erste Philosophie (1923-1924), Cartesianische Meditationen (1929) y Die Krisis der europäischen Wissenschaften und die transzendentale Phänomenologie (1935). En este sentido, puede afirmarse que Husserl continúa elaborando su doctrina de la reducción durante toda su vida.

Para comprender el recorrido husserliano y, finalmente, el auténtico sentido de la reducción, es fundamental distinguir la noción de epoché de la de reducción. La epoché se identifica con un primer momento negativo, propio de un gesto de distanciamiento crítico, que, si bien hunde sus raíces en la duda escéptica, se distancia de ella, pues propone -como su etimología lo indicaun "echarse para atrás" (Zurückhaltung), pero que tiene por objeto poder mirar lo fundamental, que antes no era visible. Practicar la epoché implica un "poner fuera de juego" (außer Aktion setzen), un "desconectar" (ausschalten), un "poner entre paréntesis" (Einklammerung) ${ }^{10}$ ciertos supuestos. Explica Husserl en Ideen I:

El mundo entero, puesto en la actitud natural, con que nos encontramos realmente en la experiencia, tomado plenamente 'libre de teorías', tal como se tiene real experiencia de él, como consta claramente en la concatenación de las experiencias no vale para nosotros ahora nada; sin ponerlo a prueba, pero también sin discutirlo, debe quedar colocado entre paréntesis. De igual modo deben sucumbir al mismo destino todas las teorías y ciencias que se refieren a este mundo, por estimables que sean y estén fundadas a la manera positivista o de cualquier otra. ${ }^{11}$

La epoché consiste, pues, en una suspensión de las creencias y teorías que operan como presupuestos de nuestra "tesis general" ingenua, que considera al mundo como independiente de nuestras operaciones de

10 Husserliana, Vol. III : Ideen zu einer reinen Phänomenologie undphänomenologischen Philosophie. Erstes Buch. Allgemeine Einfubrung in die reine Phänomenologie, Den Haag, Martinus Nijhoff, 1976, p. 63 [Hua III.1, p. 63].

11 Ibid., p. 66. 
conciencia. Cabe destacar que Husserl señala la imposibilidad de una universalización absoluta de esta "puesta entre paréntesis", pues se trata de dirigir la mirada a la esfera de la conciencia:

con buenas razones limitamos la universalidad de esta epoché. Pues si fuera tan amplia como en general puede ser, dado que puede modificarse con plena libertad toda tesis o todo juicio, o colocarse entre paréntesis toda objetividad susceptible de ser sujeto de un juicio, no quedaría dominio alguno de juicios no modificados, ni mucho menos de ciencia. Pero nuestros designios se enderezan, justamente, a descubrir un nuevo dominio científico, y un dominio tal que se conquiste justo por medio del método del colocar entre paréntesis, pero solo de un método muy precisamente limitado. ${ }^{12}$

Este punto es clave para entender la crítica de Patočka y la modalidad en que funciona la epoché radicalizada en Marion. La epoché husserliana es propuesta como un "método limitado", que no tiene un verdadero alcance universal, pues la tesis del cogito no es puesta en cuestión. Volveré sobre esta cuestión en el próximo apartado.

Ahora bien, ese momento negativo de la epoché necesita de un momento positivo: el momento de la reducción propiamente dicho. Como bien destaca Javier San Martín, la relación entre la epoché y la reducción permite distinguir dos sentidos de reducción presentes en la obra de Husserl, que son analizados en la Krisis. 1) El primer sentido se relaciona con la idea de "limitación" (Einschrankung). La reducción consiste, en este caso, en un movimiento en el que se hace epoché respecto de algo para obtener un "residuo". Según San Martín, es posible distinguir tres variantes de esta concepción de la reducción como limitación: ${ }^{13}$ 1) Reducción como epoché del mundo externo, limitándonos al mundo interno de la vida mental del sujeto. 2) Reducción como limitación a la esfera egológica. Para esta concepción, los problemas sociales serían problemas extrafenomenológicos. 3) Reducción como restricción de la investigación a las estructuras y relaciones esenciales. Este

12 Ibid., p. 65. Cfr. también Die Idee der Pbänomenologie. Fünf Vorlesungen, Den Haag, Martinus Nijhoff, 1950, p. 29 [Hua II, p. 29].

.$^{13}$ Cfr. San Martín, Javier, La estructura del método fenomenológico, Madrid, Universidad Nacional de Educación a Distancia, 1986, pp. 29-32. 
tipo de concepción cae en el error de confundir la reducción fenomenológica con la reducción eidética. Para realizar la reducción eidética se requiere una actitud eidética opuesta a la actitud dirigida a lo concreto fáctico. Pero esta actitud eidética, según Husserl, bien puede alcanzarse en la actitud natural, sin necesidad de acceder a la actitud fenomenológica. Es más, la actitud fenomenológica no implica la actitud eidética. Dice Husserl: "todas las objetividades que llamamos fenomenológicas son pensadas como singulares, objetividades individuales, cada fenómeno como un esto-aquí [Dies-da] individual". ${ }^{14}$ Como puede observarse, en todas estas variantes puede advertirse una contraposición entre la exterioridad del mundo frente al que opera la limitación y nuestra representación interior de él.

2) El segundo sentido de reducción es el que la entiende como una "reconducción" (Zurückführung). La reducción trascendental debe ser entendida como una reconducción, pues ella supera la dicotomía propia de la gnoseología moderna entre el mundo y su representación. Ya no se trata de poner en práctica ninguna limitación para encontrar un "residuo", sino de reconducir a la originariedad. No se trata, pues, de excluir ningún fenómeno, sino de preguntarse radicalmente -gracias a la "puesta entre paréntesis" de nuestros prejuicios naturales, efectuada por la epoché- respecto de las operaciones capaces de sostenerlos. La reducción reconduce a la fuente originaria de constitución y "dación de sentido" (Sinngebung). Marion asume esta noción de reducción, aunque encuentra un ámbito más originario que el de la conciencia: el ámbito de la donación.

\section{Heidegger: la epoché y los temples anímicos fundamentales}

En "L’idée de la phénoménologie et la problématique de la réduction", JeanFrançois Courtine sostiene que existe una operación de reducción en Sein und Zeit que conserva los rasgos de la reducción trascendental husserliana. Confrontando a los intérpretes que proponen que no hay reducción en Heidegger o que su filosofía se aparta de la fenomenología, Courtine plantea la hipótesis de que el análisis de la angustia en Sein und Zeit constituye la

${ }^{14}$ Zur Phänomenologie der Intersubjektivität. Texte aus dem Nachlass. Erster Teil: 1905-1920, Den Haag, Martinus Nijhoff, 1973. [Hua XIII, p. 168]. 
"repetición" heideggeriana de la problemática husserliana de la epoché y de la reducción trascendental. ${ }^{15}$

Por lo general -lamenta acertadamente Courtine-, cuando se interpreta el análisis heideggeriano de la angustia en Sein und Zeit no se repara en la situación sistemática de este análisis en la obra. Heidegger introduce la cuestión de la angustia en el $\ 39$, en el marco de la pregunta por la posible totalidad (Ganz̧eit) originaria del Dasein. Es a través del fenómeno de la angustia que el Dasein se da como unidad de sus determinaciones estructurales. $\mathrm{Y}$ es gracias a la angustia que se alcanza el suelo a partir del cual puede progresar la pregunta por el ser del Dasein. ${ }^{16}$ Teniendo en cuenta que el Dasein, simplemente siendo, está abierto para sí mismo. Y que ese "estado de abierto" (Erschlossenheit) se compone de un "comprender" (Verstehen) y de una "disposición afectiva" (Befindlichkeit), "¿habrá en el Dasein alguna disposición afectiva comprensora [verstehende Befindlichkeit] que lo deje abierto para sí mismo en forma eminente?". ${ }^{17}$

La respuesta, como es sabido, está dada por la angustia. Ella constituye la Grundbefindlichkeit que actúa como el modo de "estado de apertura" en el que el Dasein se lleva ante sí mismo. ${ }^{18}$ Courtine señala la importante diferencia de este planteo respecto de la "autodonación" (Selbstgegebenheit) husserliana. La analítica existenciaria del Dasein fáctico se despliega contra la supuesta evidencia del yo y del ser dado a sí mismo. ${ }^{19}$ Courtine destaca que, si bien esta vía de la analítica de la angustia es bien distinta de la de la reducción trascendental husserliana, esto no implica que ésta no siga su modelo. La angustia revela el Dasein a sí mismo. Courtine insiste en que allí se observa la "repetición del motivo del Rückgang auf das Subjekt". ${ }^{20}$ Por otra parte, el

${ }^{15}$ Cfr. Courtine, Jean-François, "L’idée de phénoménologie et la problématique de la réduction chez Heidegger" en Jean-Luc Marion y G. Planty-Bonjour (eds.), Phénoménologie et métaphysique, Paris, PUF, 1984, pp. 231-232.

${ }^{16}$ Cfr. Ibid., pp. 233-234.

17 Heidegger, Martin, Sein und Zeit, editado por F.-W. von Herrmann, 1977, en Gesamtausgabe, 2, Fráncfort del Meno, Klostermann, 1975-ss., p. 242 [GA 2, p. 242].

18 Cfr. Ibid.

${ }^{19}$ Cfr. Courtine, Jean-François, "L’idée de phénoménologie et la problématique de la réduction chez Heidegger”, p. 236.

20 Ibid., p. 238. 
fenómeno de la angustia actúa ciertamente como una suerte de "puesta entre paréntesis" del mundo en el sentido de la totalidad de los entes intramundanos. La angustia, por un lado, anula al mundo, pero, por el otro, lo recupera como la posibilidad del "mundo en cuanto tal" (Welt als solche). ${ }^{21}$

El camino heideggeriano, afirma Courtine, no es el husserliano, pero ambos comparten el objetivo de acceder a la instancia trascendental. Heidegger cuestiona la falta de facticidad en la reducción según es propuesta por Husserl. 'El 'que es' [das daß] de la facticidad jamás puede ser hallado en una intuición". 22 Por este motivo, no debe buscarse el espacio en el que se despliega el procedimiento regresivo trascendental en el análisis de las vivencias puras, ni en la esfera de la intuición pura.

Por otra parte, de este modo, Heidegger también da respuesta a la problemática de la "motivación" husserliana como ejercicio de la "libertad absoluta" [vollkommene Freibeit], ${ }^{23}$ pues la Stimmung "nos sobreviene". ${ }^{24}$

Coincidiendo con Courtine, Marion sostiene que Heidegger no abandona la reducción, sino que la radicaliza. Aunque el análisis de la angustia, propuesto por Marion, se centra -como en el caso de Patočka- en "Was ist Metaphysik?" y no en Sein und Zeit.25

Patočka entiende que Heidegger polemiza con Husserl en "Was ist Metaphysik?”. Pero su intención no es abandonar el trabajo del maestro, sino "buscar una interpretación ontológica de los logros de la fenomenología". ${ }^{26}$ Según el fenomenólogo checo, el padre de la fenomenología somete la epoché a la lógica. De ese modo, permanece preso de la relación sujeto-objeto y del idealismo trascendental. Por el contrario, el filósofo de Meßkirch pone en cuestión a la lógica, pues indaga profundamente en la posibilidad de la epoché.

${ }^{21}$ GA 2, p. 248.

${ }^{22}$ Ibid., p. 180.

${ }^{23}$ Hua III.1, p. 62.

24 "Die Stimmung überfällt" (GA 2, p. 182).

${ }^{25}$ En una nota al pie, Courtine destaca el análisis de la angustia y el aburrimiento propuesto por Marion en su artículo "L'angoisse et l'ennui. Pour interpréter Was ist Metaphysik ? ", Archives de Philosophie, XLIII (1980), pp. 121-146. Cfr. Courtine, JeanFrançois, "L'idée de phénoménologie et la problématique de la réduction chez Heidegger", p. 233.

26 Patočka, Jan, “¿Qué es la fenomenología?”, p. 277. 
La "tesis general" de la actitud natural es un concepto problemático, ya que presupone que la conducta primaria hacia el mundo es una tesis. Patočka destaca que Heidegger advierte que la conducta primaria hacia el mundo se da en la "esfera afectiva", en una disposición anímica desde la que se produce la apertura originaria a lo ente en su totalidad. Por lo tanto, la epoché no debe pensarse como acto teórico (¿cómo podría un acto teórico suspender un "todo" que nunca puede darse como objeto?), sino como un modo de comportarse fundado en el anonadamiento. ${ }^{27}$

En consonancia con estas ideas patočkianas, Marion ofrece en Réduction et donation un exhaustivo análisis de "Was ist Metaphysik?" y sus agregados posteriores. El hilo conductor de su lectura es el proyecto heideggeriano de acceder al fenómeno del ser. Como es sabido, Sein und Zeit constituye un intento fallido, a través de la intermediación de Dasein. Marion propone con ingenio que, en respuesta a ese primer "fracaso", en "Was ist Metaphysik?", Heidegger procura lograr el acceso de modo directo, conservando de la analítica del Dasein sólo la analítica de la angustia. Esto es posible gracias a las relecturas de su conferencia de 1929, que el filósofo de Meßkirch ensaya en el epílogo de 1943 y en la introducción de 1949. En Sein und Zeit, el ser no se da sin el ente. En la "Nachwort zu Was ist Metaphysik?", de 1943, Heidegger sostiene: “...pertenece a la verdad del ser que el ser 'se haga presente' plenamente sin el ente, pero que nunca el ente sea sin el ser". ${ }^{28}$ Según esta reinterpretación del propio Heidegger, ya en la conferencia de 1929 se considera que el ser puede darse como fenómeno prescindiendo del ente. ${ }^{29}$

Sin embargo, ¿cómo hay que entender este darse del ser si la angustia -tanto en Sein und Zeit como en "Was ist Metaphysik?"- no nos da el ser, sino la nada? Marion continúa indagando en los textos posteriores y concluye que,

27 Cfr. Ibid., p. 271. "¿En qué medida es la epojé un comportarse que anonada? En la medida en que es un no hacer uso de ninguna tesis. En ella se experimenta una libertad, una ausencia de ataduras al ente. Frente al comportarse judicativo, que no es libre, que depende del ente, se descubre una esfera en que el ente no impera, en que no fuerza a afirmar ni a negar". (Ibid., p. 269).

${ }^{28}$ Heidegger, M., Wegmarken (1919-1961), editado por F.-W. von Herrmann, 1976, en Gesamtausgabe, 9, Fráncfort del Meno, Klostermann, 1975-ss., p. 242 [GA 9, p. 306].

29 Marion, Jean-Luc, Réduction et donation. Recherches sur Husserl, Heidegger et la phénoménologie, Paris, PUF, 1989, pp. 249-252. 
en ellos, Heidegger propone una "hermenéutica de la nada" que permite establecer la equivalencia entre la nada y el ser, por medio de ciertas "acentuaciones, sustituciones y añadidos". 30

1) Las "acentuaciones" tienen por objeto interpretar la nada como el ser. En una nota agregada en 1949 se lee "la nada como 'ser". ${ }^{31}$ Asimismo, en una glosa también agregada por Heidegger con posterioridad se lee: "es decir, nada y ser son lo mismo".32 2) Por su parte, las "sustituciones" introducen la palabra "ser" donde antes decía "ente" o donde antes se había omitido. Por ejemplo, en una nota agregada en 1949 se lee: "rechazar [abweisen]: el ente para sí mismo; remitir [ver-weisen]: al ser del ente". ${ }^{3} 3$ ) Finalmente, los "añadidos" buscan establecer que la "esencia bífida" de la nada refiere a la diferencia ontológica. Por ejemplo, en un pasaje en el que Heidegger se refiere al juego de la remisión se añade que ésta refiere a la diferencia. ${ }^{34}$

Sin embargo, frente a esta "hermenéutica", Marion formula dos preguntas fundamentales: 1) ¿cuál es el hilo conductor que debe seguir esta interpretación? Y 2) ¿se debe llegar inevitablemente a este resultado?35

1) Respecto del primer interrogante, Marion encuentra una respuesta en el "Nachwort" de 1943 en el que aparece una instancia clave que no estaba presente en 1929: el Anspruch des Seins, la reivindicación del ser, la llamada del ser: "...la voz del ser [Stimme des Seins] (...) que toma como reivindicación [in den Anspruch nimmt] al hombre en su esencia a fin de que aprenda a experimentar al ser en la nada". ${ }^{36}$ Como en situación de angustia nada

30 Marion entiende que las dos referencias a la relación entre la nada y el ser, en 1929, no son suficientes para establecer la equivalencia. "En el ser del ente adviene el desistir [das Nichten] de la nada" (GA 9, p. 115) y "La nada no permanece siendo el opuesto indeterminado de lo ente, sino que se devela como perteneciente al ser del ente (...) Ser y nada se pertenecen mutuamente (...) porque el propio ser es finito en su esencia y solo se manifiesta en la trascendencia de ese Dasein que se mantiene fuera, que se arroja a la nada" (Ibid., p. 120).

31 Ibid., p. 106, n. b.

32 Ibid., p. 115, n. c.

33 Ibid., p. 114, n. a.

34 Ibid., p. 114, n. d.

${ }^{35}$ Cfr. Marion, Jean-Luc, Réduction et donation, p. 273.

36 GA 9, p. 307. 
reivindica, Marion destaca que es necesario que el ser ejerza la reivindicación guiando la interpretación. ${ }^{37}$ Es el ser mismo que llama en la angustia para que lo experimentemos en la nada.

2) Al segundo interrogante, Marion responde negativamente. Con agudeza, el fenomenólogo francés advierte que este resultado no es inevitable, pues la reinvidicación del ser exige una respuesta que puede consistir en un rechazo. ${ }^{38}$ Esta reflexión lo lleva a elaborar el que será el primer paso hacia la particularidad de su modo de concebir la reducción. Marion se pregunta por la pertinencia de la angustia como el temple anímico capaz de abrir el mundo (en sus tres dimensiones) de modo más originario. Si la llamada del ser puede ser rechazada es porque existe un "contra-existenciario" capaz de suspender la analítica existenciaria, capaz de introducir una "indiferencia ontológica". Este contra-existenciario se identifica, según Marion, con un temple más originario: el aburrimiento. En el aburrimiento, explica Marion,

el Dasein se descubre allí, pero no necesariamente para y por el ser, sino para y por una indistinción más originaria que toda indeterminación óntica: la indecisión ante la "resolución precursora" se sigue ella misma de la indecisión del ser para darse inmediatamente en un fenómeno. Formulado de otra manera: la voz sin timbre de la que el ser se reivindica ¿reivindica en nombre del ser? $\mathrm{O}$, por su silencio indistinto ¿no podría dejar aparecer un nuevo abismo, anterior, o al menos irreductible, al ser? ${ }^{39}$

El silencio, en su indistinción, parece remitir a una instancia más originaria que el ser. Esto es lo que se devela en la experiencia fundamental del aburrimiento. Marion advierte que Heidegger no examinó acabadamente en las posibilidades del "aburrimiento profundo" (tiefe Langeweile). ${ }^{40} \mathrm{El}$ aburrimiento no debe ser limitado a la "función subsidiaria" de ubicarnos ante

${ }^{37}$ Marion, Jean-Luc, Réduction et donation, p. 278.

${ }^{38}$ Cfr. Ibid., p. 282.

${ }^{39}$ Ibid., p. 283.

${ }^{40}$ Cabe señalar que Heidegger se detiene en un análisis in extenso del temple anímico del aburrimiento en su curso del semestre de invierno (1929-1930). Sin embargo, este estudio heideggeriano tampoco va más allá del horizonte del ser. Cfr. Die Grundbegriffe der Metaphysik. Welt - Endlichkeit - Einsamkeit (WS 1929/30), Gesamtausgabe, editado por F.-W. von Herrmann, 1983 [GA 29/30]. 
el ente en su conjunto, sino que se debe indagar en su capacidad más propia: la de liberarnos de la llamada del ser. ${ }^{41}$

Siguiendo a Pascal, ${ }^{42}$ Marion sostiene que el aburrimiento determina originariamente a la condición humana, pues implica la posibilidad extrema de separación respecto de su onticidad. El aburrimiento es aún más radical que una actitud nihilista, pues no busca instaurar nuevos valores, sino que permanece en una suspensión que no evalúa, no afirma ni niega. En el aburrimiento no hay antagonista.

El aburrimiento deja en su lugar al ente, sin negarlo, despreciarlo o sufrir su asalto ausente [como en el caso de la angustia]. Deja al ente en su lugar, sin afectarlo, y sin, sobre todo, dejarse afectar por él; abandona al ente pacífica y serenamente a sí mismo, como si fuera nada. Pero este abandono mismo lo define: considerando la interpelación muda del ente, de lo otro, incluso del ser, se sustraería a ello con una constancia igualmente muda. Ninguna admiración lo pone nunca en éxtasis de sí mismo. El aburrimiento neutraliza el esplendor de toda llamada, sea cual fuere; se pone a cubierto, evita exponerse, desarma el conflicto desertando del campo. Ausente de los entes, del otro, incluso del ser, no es para nadie, al punto que, en un sentido, aquel que cede al aburrimiento ya no es. ${ }^{43}$

El radical desinterés del aburrimiento nos expone a una indiferencia óntica. Ese es su poder. En el aburrimiento, desaparece el yo y con él los entes del mundo o, mejor dicho, permanecen allí, pero teñidos de vanidad. ${ }^{44}$

Ahora bien, Marion se pregunta si el aburrimiento tiene efectivamente un alcance ontológico. ${ }^{45} \mathrm{Y}$ sostiene que se puede responder afirmativamente, pues existen dos puntos donde es posible anclar el aburrimiento en el ente privilegiado ontológicamente. El primer punto es la resolución o, mejor dicho, la posibilidad de la resolución. Marion afirma que el hecho de que la impropiedad (Uneigentlichkeit) del Dasein sea posible implica negar que la

${ }^{41}$ Cfr. Marion, Jean-Luc, Réduction et donation, pp. 283-284.

42 Cfr. Pascal, Blaise, Pensées, L. Lafuma ed., Paris, Seuil, 1963, J\ 24, 136, 941 y 919.

43 Marion, Jean-Luc, Réduction et donation, pp. 286-287.

44 Cfr. Ibid., pp. 287-288. Y será este punto, esta vanidad, la que llevará a Marion a dar el último paso hacia la reducción erótica. Volveré sobre esta cuestión.

45 Cfr. Ibid., p. 290. 
remisión al ser constituya la posibilidad última de lo que soy. Si la impropiedad define tan esencialmente al Dasein como la propiedad, entonces por medio de la indecisión del aburrimiento es posible liberarse del destino de Dasein. El segundo punto es el "tener que ser" propio del Dasein. Este "tener que ser" que define también el modo de ser del Dasein implica que éste tiene que ser su ser, pero también que puede no serlo. ${ }^{46}$ Es en estos aspectos del Dasein donde puede radicar el aburrimiento.

Sin embargo, esta vanidad de la que se tiñe el mundo en el aburrimiento no es más que la contracara negativa del temple anímico fundamental de la filosofía de Marion: el amor. ${ }^{47}$ En Le phénomène érotique, Marion da cuenta de cómo es posible dar este paso final afirmativo a partir de la constatación de la vanidad de la certeza objetual del cogito. "La certeza de mi existencia no basta para volverla justa, ni buena, ni bella, ni deseable, es decir, no basta para asegurarla". ${ }^{48}$ La certeza no alcanza para darle un sentido a mi existencia. En este punto, Marion propone que la pregunta decisiva, la única pregunta que puede concernirnos realmente y escapar a la vanidad, es la siguiente: "¿me aman?" (m'aime-t-on ?). Sólo el ser amados puede asegurar nuestra existencia, puede darle un sentido, puede volverla justa, buena, bella y/o deseable. La indiferencia de la simple existencia resulta insoportable, insostenible: nadie en su sano juicio puede conformarse con el mero hecho de existir. "Renunciar a plantear(se) la pregunta '¿me aman?', y sobre todo a la posibilidad de una respuesta positiva, implica nada menos que renunciar a lo humano en uno mismo". 49

A partir la aceptación de esta pregunta se entra en el régimen de reducción erótica, que se despliega en diversas figuras. Luego de indagar en la insuficiencia del amor a sí mismo y de la vía del intentar "hacerse amar", 50 Marion propone cambiar el punto de partida. La pregunta "¿me aman?"

\footnotetext{
${ }^{46}$ Cfr. Ibid., pp. 291-293.

47 "Lecho vacío de un amor ausente, el mundo se ofrece desolado a la mirada del aburrimiento, cuya impotencia para la caridad tan solo vierte vanidad: el reverso de la caridad" (Marion, Jean-Luc, Dieu sans l'être, Paris, Fayard, 1982, p. 188).

48 Marion, Jean-Luc, Le phénomène érotique, Paris, Grasset, 2003, p. 42.

49 Ibid., p. 39.

50 “Quien quiera hacerse amar llega a odiarse a sí mismo, luego odiará a cualquier otro distinto de sí y finalmente se inscribirá en el odio de todos hacia todos” (Ibid., p. 111).
} 
parece no arriesgar demasiado, parece esperar una reciprocidad. El ego que busca su seguridad tiene una comprensión estrecha del amor, pues espera un intercambio. Pero la reciprocidad no es un rasgo del amor. ${ }^{51}$ Marion plantea entonces la necesidad de radicalizar la reducción erótica para superar la exigencia de reciprocidad y la presuposición de una seguridad que debería advenirme. Es necesario hacerse otra pregunta: “¿puedo amar, yo primero?” (puis-je aimer, moi le premier?), 52 es necesario un desplazamiento desde la figura del amado a la figura del amante.

$\mathrm{El}$ amante que toma la iniciativa de amar toma la iniciativa (actividad) de entregar la iniciativa (pasividad), ${ }^{53}$ de exponerse sin reserva a un otro y a lo otro que acontece sin poder ser previsto. Su decisión es la decisión de ya no decidir, su acción es la acción de ya no actuar; y su devenir un sí mismo se alcanza, no en un gesto de auto-posicionamiento o apropiación de sí, sino por el contrario en una operación de desapropiación, en una exposición que presupone y ocurre por otro (aun cuando este otro no responda a ese amor). El avance del amante, el decidirse a amar amar, confirma paradójicamente su dependencia respecto de otro. ${ }^{54}$ En el amor se devela la imbricación entre

51 "La reciprocidad fija la condición de posibilidad del intercambio, pero ella atesta también la condición de imposibilidad del amor” (Ibid., p. 115).

52 Ibid., p. 116.

${ }^{53}$ Cfr. Marion, Jean-Luc, Étant donné. Essai d'une phénoménologie de la donation, seconde édition corrigé, Paris, PUF, 1998, p. 15.

54 "Paradójicamente, no aparezco cuando me decido yo mismo por y para mí mismo, sino cuando me decido por otro, porque entonces él puede confirmar que yo soy: decidiéndome por él, es por él que yo aparezco. En mi decisión por otro, mi decisión de amar por avance un otro que yo, se decide mi fenomenicidad más propia. No decido acerca de mí mismo por mí mismo, sino por la mirada del otro; no por una resolución precursora sin testimonio ni lugar, sino por el amor de avance, en la distancia en que me expongo al otro (\$37). La decisión de amar sigue siendo entonces válida, aun cuando yo no realice efectivamente el amor por avance, con tal de que me resuelva a ello; con tal de que formalmente al menos me decida a decidirme. Hacer el amor por anticipado quizás no dependa de mí, pero decidirme a decidirme sí. Amar por avance tal vez no dependa de mí, pero amar amar (amare amare) sí. Nada puede separarme de mi libertad de hacerme amante" (Marion, Jean-Luc, Lephénomène érotique, p. 148). Cabe señalar que las figuras de la reducción erótica continúan desplegándose 
pasividad y actividad propia del carácter receptivo del adonado. Al decidirme a amar amar, me decido activamente a devenir pasivo en una entrega al otro. En este sentido, si bien el libro propone una descripción del fenómeno erótico, es decir, del amor como fenómeno, cabe advertir que -aun cuando el autor no lo haga explícito- el amor es el temple anímico fundamental que actúa como epoché en la reducción erótica como modalidad extrema de la tercera reducción..$^{55} \mathrm{El}$ amor desplaza al temple paralizante de la angustia, precisamente porque consiste en una tonalidad afectiva que combina un componente activo con otro pasivo, y de ese modo, permite la reconducción más acabada a la donación, ya que opera una apertura absoluta e incondicionada a lo dado. Esta apertura ya no se da desde la mera indiferencia, sino que logra dar un paso positivo hacia un involucramiento activo en primera persona que consiste en aceptar la más completa entrega pasiva. Esto permite una cabal fenomenalización, no solo del adonado, sino de todo fenómeno según los términos de su donación. Como bien señala Emmanuel Housset: "Desde L'idole et la distance a Étant donné y hasta Certitudes négatives, [Marion] no ha dejado de describir el ser del amor, menos como un fenómeno que se constituiría en un horizonte, que como un poder de fenomenalización". ${ }^{6}$

hasta encontrar en Dios al primer amante, que hace posible el avance del amante porque siempre ya hemos sido amados por él (cfr. Ibid., p. 341).

${ }^{55}$ Marion aclara la relación entre la reducción erótica y la tercera reducción en el "Prólogo a la edición española" de Étant donné. La reducción erótica no constituye una cuarta reducción que viene a reemplazar o a superar la tercera reducción, sino que se despliega como la posibilidad más extrema de la tercera reducción, aquella que proviene del cruce de los amantes y ya no del encuentro con un fenómeno mundano. Cfr. Marion, Jean-Luc, "Prólogo a la edición española", en Marion, Jean-Luc, Siendo dado. Ensayo para una fenomenología de la donación, trad. J. Bassas Vila, Madrid, Síntesis, 2008, p. 14.

${ }^{56}$ Housset, Emmanuel, "Préface. L'être de l'amour" en Tabet, Pascale, Amour et donation chez Jean-Luc Marion. Une phénoménologie de l'excès, Paris, L'Harmattan, 2017, p. 9. Este pasaje de la donación a la fenomenalización nos remite a la operación propia de la hermenéutica, según es definida por Marion en Reprise du donné: "La hermenéutica gestiona la distancia entre lo que se da y lo que se muestra, interpretando la llamada (o, frecuentemente, la intuición) por la respuesta (concepto o significación)" (Marion, JeanLuc, Reprise du donné, p. 89). Para un análisis de una posible "hermenéutica del amor" 


\section{Patočka: epoché y reducción}

Luego de constatar el carácter ontológico del aburrimiento, Marion propone, en Réduction et donation, su tercera reducción. El carácter más originario del aburrimiento ante la angustia no busca negar la posibilidad de un ser propio del Dasein, sino, por el contrario, como ya hemos explicado, procura develar una instancia más originaria que el ser, a la que el "ahî" podría exponerse. ${ }^{57}$ Se trata de seguir los pasos ya dados por Emmanuel Lévinas, pero ir más allá. La llamada del otro sustituye a la llamada del ser porque se prueba más originaria que ésta. Pero, entonces -reflexiona agudamente Marion-, la instancia originaria es la llamada en tanto tal, la llamada pura, con prescindencia de si ésta es ejercida en beneficio de esta o aquella autoridad. ${ }^{58}$ La estructura de la llamada es más originaria que la llamada del ser o que la llamada del otro. Es posible reconducir la reivindicación del ser a la llamada pura: puede reducirse toda reivindicación a la forma pura de la llamada. "Luego de la reducción trascendental y la reducción existencial, interviene la reducción a y de la llamada. Lo que se da solo se da a aquel que se entrega [s'adonne] a la llamada bajo la forma pura de una confirmación de la llamada reproducida en tanto que recibida". ${ }^{9}$

De este modo, Marion presenta su tercera reducción: la reducción a la forma pura de la llamada, que tiene la particularidad de contener y, a la vez, en cierto sentido, superar a las otras dos reducciones (trascendental y existencial). ${ }^{60}$

en la obra marioniana, me permito remitir a mi Hermenéutica del amor. La fenomenología de la donación de Jean-Luc Marion en diálogo con la fenomenología del joven Heidegger, Buenos Aires, Editorial SB, colección Post-Visión, 2019.

${ }^{57}$ Cfr. Marion, Jean-Luc, Réduction et donation, p. 294.

${ }^{58}$ Cfr. Ibid., pp. 295-296.

${ }^{59}$ Ibid., p. 296.

${ }^{60}$ En palabras de Marion: "La tercera reducción retoma, valida y descalifica a la vez a las dos primeras" (Marion, Jean-Luc, "Prólogo a la edición española", p. 14). Según Marion, esto es así porque el esquema de las tres reducciones repite la modalidad de relación entre los tres órdenes pascalianos. "La distinción entre el orden del cuerpo, el orden del espíritu y el orden del corazón, en una jerarquía en la que cada orden superior ve a los órdenes inferiores sin que éstos puedan verlo, nos conduce (con 
La llamada aparece de este modo como el esquema originario [schème originaire] de las dos reducciones anteriores, precisamente porque solo ella permite reconducir a..., en lo que se exige entregarse a lo dado de la llamada como tal [s'adonner à la donne de l'appel]: rendirse a la llamada [se rendre à l'appel, en el doble sentido de abandonarse a ella y de desplazarse hacia ella. En tanto que pura reducción -dado que es perfecta reconducción a...-, la llamada que reivindica para sí misma incumbe eminentemente a la fenomenología. ${ }^{61}$

La tercera reducción constituye pues el núcleo inadvertido de las reducciones anteriores. En este sentido, la reducción a la forma pura de la llamada no deslegitima a la reducción trascendental ni a la reducción existencial, pero sí las reubica en relación con el carácter originario y más amplio que la reducción marioniana ostenta, explicitando los límites de sus respectivos campos de aplicación.

En Étant donné, Marion retoma este esquema de la triple reducción planteando la necesidad de superar el horizonte de la objetidad -postulado por la reducción husserliana- y el horizonte de la enticidad -postulado por la reducción heideggeriana-. Según Marion, sólo la tercera reducción, entendida como una reducción a la donación, es capaz de superar las limitaciones del objeto y del ser, pues la donación es la "figura extrema de la fenomenicidad" que "precede y rebasa cualquier otra especificación". ${ }^{62}$

Finalmente, en Le phénomène érotique, Marion explora la posibilidad última de la tercera reducción a partir de la reducción erótica, que ocurre cuando el adonado se recibe ya no de un fenómeno mundano, sino de otro adonado. Como ya hemos señalado, en el "Prólogo a la edición española" de Étant donné, Marion es claro respecto de cómo debe ser entendida la relación

todas las aproximaciones necesarias) a las reducciones distinguidas por Reducción y donación y puestas por obra posteriormente en Siendo dado" (Ibid., pp. 13-14). Marion también refiere explícitamente al modelo pascaliano en la entrevista "Réponses à quelques questions" (cfr. Marion, Jean-Luc, "Réponses à quelques questions", Revue de Métaphysique et de Morale. "À propos de Réduction et donation de Jean-Luc Marion", 96, 1 (1991), p. 66).

${ }^{61}$ Marion, Jean-Luc, Réduction et donation, pp. 296-297.

62 Marion, Jean-Luc, Étant donné, p. 60. 
entre la tercera reducción y la reducción erótica: "Ésta ni se añade a la tercera reducción (a lo dado) ni se confunde con ella, sino que la radicaliza conduciéndola a su posibilidad más extrema". ${ }^{63}$

Ahora bien, ¿cómo entiende Marion que opera la "perfecta reconducción" de la tercera reducción? En diversos pasajes de su obra aparece una suerte de identificación de la reducción con la epoché. En Étant donné, por ejemplo, Marion define la reducción en los siguientes términos: "La reducción opera por excelencia de esta manera: suspende las 'teorías absurdas', las falsas realidades de la actitud natural, el mundo objetivo, etc., para dejar que las vivencias dejen aparecer tanto como sea posible lo que se manifiesta como y por ellos; su función culmina al despejar los obstáculos para la manifestación". ${ }^{64}$ Puede observarse allí cómo Marion identifica la reducción con su núcleo: la epoché. 65 Es la epoché la encargada de "suspender las teorías absurdas" y "las falsas realidades de la actitud natural", y de este modo, es ella la encargada de "despejar los obstáculos para la manifestación". Pero, entonces ¿cómo debe entenderse esta equiparación sin más entre epoché y reducción? ¿Marion no las distingue?

En 2015, en su artículo "Monde et donation. Une révision du quatrième principe de la phénoménologie", apoyándose en la fenomenología patočkiana, Émilie Tardivel propone una lectura de la tercera reducción. Según la autora, es posible pensar la reducción en Marion como una patočkiana "universalización de la epoche" ${ }^{66}$

En “Epoché y reducción”, Patočka se pregunta qué sucedería si la epoché no se detuviese ante la tesis del sí mismo propio, sino que fuese aplicada de modo universal. El checo propone reflexionar respecto de lo que ocurriría si no se utilizara de "manera automática" la tesis del cogito, sino que también se

\footnotetext{
63 Marion, Jean-Luc, "Prólogo a la edición española", p. 14.

${ }^{64}$ Marion, Jean-Luc, Étant donné, p. 16.

${ }^{65}$ Cfr. Patočka, Jan, “¿Qué es la fenomenología?”, p. 272.

66 Tardivel, Émilie, "Monde et donation”, p. 124. Cabe destacar que es el propio Marion, en su artículo "La donation, dispense du monde", quien sugiere un posible vínculo de su fenomenología con la de Patočka (cfr. Marion, Jean-Luc, "La donation, dispense du monde", Philosophie, 118, 2 (2013), pp. 78-90). Las ideas de este artículo constituyen el capítulo tercero de Reprise du donné, "La donation, ou la dispense du monde" (cfr. Marion, Jean-Luc, Reprise du donné, pp. 99-146).
} 
pusiera a esta última tesis entre paréntesis. Patočka concluye que "entendida así, la epoché no es un acceso a ningún ente o pre-ente, sea mundano o nomundano, pero justamente por ello es quizás el acceso al aparecer en lugar de lo que aparece, esto es, al aparecer en tanto tal". ${ }^{67}$

Siguiendo a Patočka, Tardivel propone que lo que realmente es radicalizado por Marion no es la reducción, sino la epoché. La tercera reducción, que ya no reduce ni a la conciencia ni al ser ni a ninguna instancia regional, conduce a los mismos resultados que la epoché patočkiana al reconducir al aparecer mismo, a la doneidad de lo dado, ${ }^{68}$ a la donación. En palabras de Tardivel: "La reducción a la llamada ofrece así la paradoja de una reducción que no reduce, e incluso muestra, a su vez, que toda reducción no reduce, sino que amplía y da". ${ }^{69}$

Es más, si se entiende la tercera reducción como una epoché es posible responder a la objeción de Romano respecto de la imposibilidad de conciliar en la obra de Marion un método decididamente subjetivo, como la reducción, con un tema radicalmente asubjetivo. Si bien es cierto que la reducción es un procedimiento subjetivo, cabe preguntarse si puede decirse lo mismo de la epoché. Según Patočka, el acto de libertad que implica la epoché se basa en algo

${ }^{67}$ Patočka, Jan, “Epoché y reducción”, p. 247.

68 Jean-François Lavigne propone que el término Gegebenheit debería haber sido traducido por Marion como donéité y no como donation, para enfatizar de modo más explícito el mero hecho de lo dado: "la Gegebenheit husserliana designa el estatuto de lo que ya se encuentra dado, que no se confunde con el proceso de la (Sinn-) Gebung. La objetivación reflexiva que efectúa la mirada fenomenológica en Husserl siempre re-aprehende lo dado, ya sea primario (por ejemplo, el datum hylético en curso de constitución), ya sea originario (la impresión originaria del presente viviente), antes de su donación: en su estar-(ya)-dado, o en su "doneidad" (Gegebenheit)" (cfr. Lavigne, Jean-François, Husserl et la naissance de la phénoménologie, 1909-1913, Paris, PUF, 2003, p. 25, n. 1). Marion parece asumir la crítica cuando utiliza el término donéité en Figures de phénoménologie: "Nuestra intención sólo consiste aquí en verificar el estatuto estrictamente filosófico, y también fenomenológico, incluso pre-fenomenológico de la donación, consiste, por tanto, en comprenderla como una modalidad de la fenomenicidad y no como algo dado óntico, como una doneidad (Gegebenheit) y no como una fundación metafísica u ontoteológica" (Marion, Jean-Luc, Figures de phénoménologie : Husserl, Heidegger, Lévinas, Henry, Derrida, Paris, Vrin, 2012, p. 28).

69 Tardivel, Émilie, "Monde et donation”, p. 125. 
distinto a la libertad del sujeto, pues, "¿cómo sería posible un comportarse de esta índole, una libertad tal, si a su base no hubiese una experiencia de la posibilidad fundamental de distanciarnos de toda actividad, incluida la judicativa?". ${ }^{70}$ La epoché, a diferencia de la reducción, no consiste en un procedimiento completamente subjetivo, sino que deviene posible gracias a una experiencia previa que es de carácter asubjetivo. Esta experiencia asubjetiva, indisponible, no es otra que la Grunderfahrung heideggeriana, la experiencia fundamental que se da en los temples anímicos señalados (aburrimiento y amor). De este modo, es posible resolver la paradoja señalada por Romano de una reducción operada por el propio fenómeno. La reducción a la llamada pura reduce el yo a una pasión. ${ }^{71} \mathrm{La}$ auto-reducción es posible gracias a la reducción del sujeto a una asubjetividad. ${ }^{72}$

En Reprise du donné, Marion entiende que es posible responder a la lectura de Tardivel, señalando que no deben oponerse la reducción y la epoché, sino que -siguiendo a Jérôme de Gramont (que, a su vez y paradójicamente, también sigue a Patočka)- debe entenderse a la epoché como aquello que permanece en toda reducción dándole su univocidad. Dice Gramont: “...la pluralidad de nombres de la reducción no impide su imantación hacia un primer nombre que constituye su figura última". ${ }^{73}$ Esta figura última es la epoché. Retomando esta idea, Marion sostiene: "Por tanto, la epoché no se confunde con la reducción, pero precisamente porque ella conserva, en cada uso que se hace de ella, una univocidad indeterminada. Hay que admitir la pluralidad de reducciones precisamente porque ellas ponen en práctica la única epoche" ${ }^{74}$ Marion nos advierte aquí: 1) que él distingue con claridad la

70 Patočka, Jan, “¿Qué es la fenomenología?”, pp. 269-270.

${ }^{71}$ Cfr. Marion, Jean-Luc, Réduction et donation, pp. 290-291. Tardivel sostiene -dando respuesta a la objeción de Michel Henry respecto de un formalismo en la reducción a la llamada pura- que "la indeterminación de la llamada no se distingue de la pasión que ella pone en práctica (...) Lejos de vaciar el aparecer de todo contenido concreto, la forma pura de la llamada le da una 'materia fenomenológica impresional"' (Tardivel, Émilie, "Monde et donation", p. 127).

72 Cfr. Ibid., pp. 127-128.

73 De Gramont, Jérôme, "Le pluriel des réductions et l'unique époche”, Archivio di Filosofia, 83 (2015), p. 75.

74 Marion, Jean-Luc, Reprise du donné, p. 54. 
epoché de la reducción y 2) que no es necesario optar entre una u otra, sino que ambas se articulan de un modo esencial.

Marion concluye en este último texto que la relación entre epoché y reducción debe ser entendida en los siguientes términos:

La reducción sigue un método, es decir que ella toma prestado, en todas sus puestas en práctica, el camino indicado por la epoché, de tal modo que, en todo momento de la actualización de la donación, la reducción practica aun y siempre la suspensión, la retención y la puesta entre paréntesis de lo que no se encuentra aun absolutamente dado (...) La epoché no rivaliza con la reducción, sino que la sirve. ${ }^{75}$

Marion sostiene que la reducción, en sus diversas modalidades, se sirve de la epoché como un ministro; se da una "relación ministerial" entre ellas. ${ }^{76}$ Ahora bien, la respuesta de Marion -que asienta su posición última al respecto- no parece suficiente. Llama la atención este intento de distanciamiento, cuando un año antes, Marion acepta sin más la sugerencia de Tardivel como válida. En sus "Réponses" a las lecturas de su obra presentadas en el coloquio "La philosophie de Jean-Luc Marion: sources et débats" celebrado en el Collège des Bernardis, el 23 de enero de 2013, y cuyas intervenciones fueron publicadas en 2015- luego de explicar la ausencia de un donador originario, único y trascendental, y de señalar la función de los adonados respecto de la recepción de la donación y su fenomenalización, Marion sostiene: "En este caso ¿no convendría comprender la tercera reducción, en su sentido a-subjetivo (sin sujeto trascendental), a la manera de Patočka, y hablar más bien de epoché; de la donación? Una comentadora [Marion cita en una nota al pie el artículo de Émilie Tardivel] acaba de hacer esta sugerencia, me parece que quizás podríamos tomarla". ${ }^{77}$

Por otra parte, la propuesta de Tardivel también encuentra respaldo en el libro de 2014 sobre Courbet. Allí, siguiendo a Patočka, a fin de explicar el

\footnotetext{
75 Ibid., p. 55.

76 Cfr. Ibid., p. 56.

77 Marion, Jean-Luc, "Réponses", en Philippe Capelle-Dumont (éd.), Philosophie de Jean-Luc Marion. Phénoménologie, théologie, métaphysique, Paris, Hermann, 2015, pp. 128129.
} 
operar propio del artista, Marion sugiere que éste pone en práctica una "epoché sin reducción":

Mientras que la reducción, según es habitualmente definida por Husserl, sólo pone entre paréntesis la región-mundo reforzando la certeza de la región-conciencia, la epoché suspende el primado de este ego exponiéndolo directamente a lo dado anterior del mundo, que no constituye más la colección estática, eventualmente total y totalizante, sino que surge como un acontecimiento. ${ }^{78}$

La verdadera creación artística no parte de la voluntad del artista, sino de la "neutralización" de su ego por medio de la epoché sin reducción que le permite entregarse al acontecer imprevisible de la obra. El ámbito del arte parece ofrecer la posibilidad de una fenomenología sin reducción. Pero entonces, ¿cómo conciliar la defensa marioniana de la reducción y esta nueva vía de la epoché sin reducción ensayada en esta obra?

La respuesta se encuentra entre ambas posiciones (la de Courbet y la de Reprise du donné). Efectivamente, Marion radicaliza la epoché al modo patočkiano, pero esto no lo lleva a prescindir de la reducción. Marion distingue la epoché de la reducción según las indicaciones husserlianas. La epoché constituye el momento crítico negativo en el que se suspenden "las teorías absurdas" y la reducción se identifica con el momento positivo de reconducción a lo originario. Ahora bien, la epoché opera de un modo tan radical que se superpone con la reducción, pues la reconducción a lo originario se da en el gesto mismo de suspensión: la epoché "abre y da" porque es su misma "puesta entre paréntesis" la que nos conduce a la instancia más originaria, a la donación. La tercera reducción es el núcleo inadvertido de las reducciones anteriores (trascendental y existencial) porque en ella se da una reducción absoluta de la reducción a su núcleo: la epoché.

\section{Conclusión: la reducción en la fenomenología de la donación}

Para entender cómo opera la reducción en la fenomenología de la donación es necesaria la explicitación que se llevó a cabo en este artículo. Como hemos demostrado, la tercera reducción se caracteriza por dos rasgos decisivos. En

${ }^{78}$ Marion, Jean-Luc, Courbet ou la peinture à l’cil, Paris, Flammarion, 2014, pp. 94-95. 
primer lugar, Marion sigue el modelo heideggeriano de reducción al transformar a la epoché en una Grundstimmung. El temple anímico fundamental, sin embargo, no es la angustia, ni el aburrimiento en el horizonte del ser, sino, en primer término, un aburrimiento entendido en su radicalidad, capaz de operar una indiferencia ontológica. Y, finalmente, el amor como la tonalidad que abre de modo más acabado a la donación. Es decisivo advertir que la centralidad del amor en la propuesta marioniana también se juega en la función que esta tonalidad desempeña como la epoché de la reducción érotica en tanto que modalidad más propia de la tercera reducción.

En segundo lugar, Tardivel acierta al señalar que Marion radicaliza el alcance de la epoché. La tercera reducción actúa como una reducción radical, pues es reducida a su núcleo: la epoché. Y, a su vez, ese núcleo es liberado en el sentido patočkiano de su universalización. Pero, para radicalizar la operación de la epoché en un sentido extremo, es preciso que esa disposición afectiva ya no sea la angustia (que reconduce a la nada, al ser o incluso al mundo en el sentido patočkiano). El temple anímico reductor debe ser capaz de poner entre paréntesis también a la diferencia ontológica y reconducir de modo activo/pasivo a la donación (aburrimiento/amor).

Marion sostiene que existe una "relación ministerial" entre reducción y epoché. Sin embargo, también destaca que la reducción sigue el "camino indicado por la epoche". Habría que decir, pues, que la particularidad de la noción marioniana de la reducción es que ella se solapa sin más con la epoché. La reducción marioniana opera por el gesto mismo de la epoché, pues ésta es tan radical, que, lejos de excluir, limitar o restringir, da ilimitadamente al ofrecer un acceso al estadio que ya no excluye nada porque no presupone ningún modo de darse. La "perfecta reconducción" es la reconducción a la donación ejecutada por la epoché radicalizada.

\section{Bibliografía}

- Bernet, Rudolf, La vie du sujet. Recherches sur l'interprétation de Husserl dans la phénoménologie, Paris, PUF, 1994. 
- Courtine, Jean-François, "L'idée de phénoménologie et la problématique de la réduction chez Heidegger" en Jean-Luc Marion y G. Planty-Bonjour (eds.), Phénoménologie et métaphysique, Paris, PUF, 1984.

- De Gramont, Jérôme, "Le pluriel des réductions et l'unique époche", Archivio di Filosofia, 83 (2015).

- Fink, Eugen, "Die phänomenologische Philosophie Edmund Husserls in der gegenwärtigen Kritik", Kant-Studien, 38, 1-2 (1933).

- Heidegger, Martin, Sein und Zeit, editado por F.-W. von Herrmann, 1977, en Gesamtausgabe, 2, Fráncfort del Meno, Klostermann, 1975-ss.

- Heidegger, Martin, Wegmarken (1919-1961), editado por F.-W. von Herrmann, 1976, en Gesamtausgabe, 9, Fráncfort del Meno, Klostermann, 1975-ss.

-Heidegger, Martin, Die Grundbegriffe der Metaphysik. Welt-Endlichkeit - Einsamkeit (WS 1929/30), editado por F.-W. von Herrmann, 1983, Gesamtausgabe, 29/30, Fráncfort del Meno, Klostermann, 1975-ss.

- Henry, Michel, "Quatre principes de la phénoménologie”, Revue de Métaphysique et de Morale. À propos de Réduction et donation de Jean-Luc Marion, 96, 1 (1991).

- Housset, Emmanuel, "Préface. L'être de l'amour" en Tabet, Pascale, Amour et donation chez Jean-Luc Marion. Une phénoménologie de l'excès, Paris, L'Harmattan, 2017

- Husserl, Edmund, Husserliana, Vol. II : Die Idee der Phänomenologie. Fünf Vorlesungen, Den Haag, Martinus Nijhoff, 1950.

- Husserl, Edmund, Husserliana, Vol. XIII: Zur Pbänomenologie der Intersubjektivität. Texte aus dem Nachlass. Erster Teil: 1905-1920, Den Haag, Martinus Nijhoff, 1973.

- Husserl, Edmund, Husserliana, Vol. III: Ideen zu einer reinen Phänomenologie undphänomenologischen Philosophie. Erstes Buch. Allgemeine Einfubrung in die reine Phänomenologie, Den Haag, Martinus Nijhoff, 1976.

- Lavigne, Jean-François, Husserl et la naissance de la phénoménologie, 1909-1913, Paris, PUF, 2003.

- Marion, Jean-Luc, "L'angoisse et l'ennui. Pour interpréter Was ist Metaphysik ?", Archives de Philosophie, XLIII (1980), pp. 121-146.

- Marion, Jean-Luc, Dieu sans l'être, Paris, Fayard, 1982.

- Marion, Jean-Luc, Réduction et donation. Recherches sur Husserl, Heidegger et la phénoménologie, Paris, PUF, 1989.

- Marion, Jean-Luc, "Réponses à quelques questions", Revue de Métaphysique et de Morale. "Á propos de Réduction et donation de Jean-Luc Marion", 96, 1 (1991).

- Marion, Jean-Luc, Étant donné. Essai d'une phénoménologie de la donation, seconde édition corrigé, Paris, PUF, 1998.

- Marion, Jean-Luc, Le phénomène érotique, Paris, Grasset, 2003.

- Marion, Jean-Luc, "Prólogo a la edición española", en Marion, Jean-Luc, Siendo dado. Ensayo para una fenomenología de la donación, trad. J. Bassas Vila, Madrid, Síntesis, 2008. 
- Marion, Jean-Luc, Figures de phénoménologie : Husserl, Heidegger, Lévinas, Henry, Derrida, Paris, Vrin, 2012.

- Marion, Jean-Luc, "La donation, dispense du monde", Philosophie, 118, 2 (2013).

- Marion, Jean-Luc, Courbet ou la peinture à l'ceil, Paris, Flammarion, 2014.

- Marion, Jean-Luc, "Réponses", en Philippe Capelle-Dumont (éd.), Philosophie de JeanLuc Marion. Phénoménologie, théologie, métaphysique, Paris, Hermann, 2015.

- Marion, Jean-Luc, Reprise du donné, Paris, PUF, 2016.

- Pascal, Blaise, Pensées, L. Lafuma ed., Paris, Seuil, 1963.

- Patočka, Jan, El movimiento de la existencia bumana, trad. T. Padilla, J. M. Ayuso y A. Serrano de Haro, Madrid, Ediciones Encuentro, 2004.

- Roggero, Jorge Luis, Hermenéutica del amor. La fenomenología de la donación de Jean-Luc Marion en diálogo con la fenomenología del joven Heidegger, Buenos Aires, Editorial SB, colección Post-Visión, 2019.

- Romano, Claude, "Remarques sur la méthode phénoménologique dans Étant donné de Jean-Luc Marion", Annales de philosophie, 21 (2000).

- San Martín, Javier, La estructura del método fenomenológico, Madrid, Universidad Nacional de Educación a Distancia, 1986.

- Tardivel, Émilie, "Monde et donation. Une révision du quatrième principe de la phénoménologie", Revue de Métaphysique et de Morale, 85, 1 (2015).

Recibido: 03/2019. Aceptado: 06/2019. 